\title{
Altered glutamate cysteine ligase activity in peripheral blood mononuclear cells from patients with systemic lupus erythematosus
}

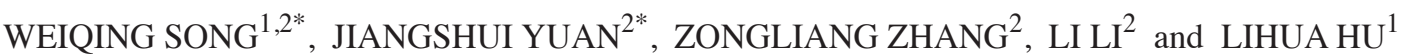 \\ ${ }^{1}$ Tongji Medical College, Huazhong University of Science and Technology, Wuhan, Hubei 430030; \\ ${ }^{2}$ Qingdao Municipal Hospital, Qingdao, Shandong 266071, P.R. China
}

Received December 11, 2013; Accepted April 1, 2014

DOI: $10.3892 /$ etm.2014.1689

\begin{abstract}
Reductions in glutathione (GSH) levels have been shown to be associated with aging and the pathogenesis of a variety of diseases, including systemic lupus erythematosus (SLE). Glutamate cysteine ligase (GCL) catalyzes the first and rate-limiting step of GSH synthesis. In order to appraise the correlation between oxidative stress and the severity and activity of SLE, GSH, oxidized GSH (GSSG) and thioredoxin (TRX) concentrations and the enzymatic activity levels of GCL in peripheral blood mononuclear cells (PBMCs) from patients with SLE and healthy controls were studied. In patients with SLE, the levels of GCL activity and GSH decreased, while TRX and GSSG levels increased when compared with those in the healthy controls. GSH concentrations and GCL activity levels negatively correlated with the SLE disease activity index and erythrocyte sedimentation rate. Furthermore, patients with SLE and nephritis had lower levels of GSH and GCL activity and higher levels of TRX and GSSG compared with those in SLE patients without nephritis. Therefore, the results of the present study indicate that insufficient levels of GSH and GCL activity in PBMCs may contribute to the pathogenesis of SLE.
\end{abstract}

\section{Introduction}

Systemic lupus erythematosus (SLE) is a multifactorial autoimmune disease. The assortment of autoantibodies produced is broad and, as a consequence, the manifestations of the disease are diverse (1). SLE is characterized by arthritis, cutaneous rash, vasculitis, involvement of the central nervous system

Correspondence to: Dr LiHua Hu, Tongji Medical College, Huazhong University of Science and Technology, 13 Hangkong Road, Wuhan, Hubei 430030, P.R. China

E-mail: songweiqing68@163.com

*Contributed equally

Key words: glutathione, glutamate cysteine ligase, peripheral blood mononuclear cells, systemic lupus erythematosus, oxidative stress
(CNS) and renal and cardiopulmonary manifestations (2). Reactive oxygen species (ROS) have been considered as risk and enhancer factors for autoimmune diseases (3). Several previous studies have indicated that the chronic immune activation present in the disease is caused by the depletion of intracellular glutathione (GSH) through oxygen-derived free radical production, known as oxidative stress (4-6).

Several clinical conditions, particularly inflammatory and/or immuno-mediated disorders, have been associated not only with oxidative stress in terms of increased ROS formation, but also with impaired antioxidant status mainly in terms of reduced GSH levels and lowered cellular redox potential (7). GSH protects against oxidative stress and detoxifies xenobiotics; thus, is involved in the maintenance of homeostasis. Therefore, reductions in GSH levels are associated with aging and the pathogenesis of a variety of diseases, autoimmune or not, including SLE, rheumatoid arthritis (RA), autoimmune thyroiditis, muscular dystrophy, amyotrophic lateral sclerosis, acquired immunodeficiency syndrome (AIDS), Alzheimer's, alcoholic liver disease, cataract genesis, respiratory distress syndrome and Werner syndrome (8-11). Altered GSH concentrations may play an important role in a number of autoimmune pathological conditions, prevalently elicited, determined and maintained by inflammatory/immune responses mediated by oxidative stress reactions (12).

In several diseases, including SLE, abnormal T-cell activation and death occurs, which is crucially dependent on the controlled production of ROS and adenosine triphosphate (ATP) in the mitochondria (13). ROS exert their prooxidant activity by inducing tissue damage and the dissociation of iron ions or iron-containing compounds (heme) from a protein-bound state. In addition, the exposure of specific antibodies to heme, transition metal ions or ROS may induce the appearance of new antigen-binding specificities for various autoantigens (14). ROS generation also provides oxidants for thiol oxidation or peroxynitrite formation, which may be a basis for antibody modification (15).

A number of studies have shown that excessive ROS production damages macromolecules, including DNA and proteins, and modulates the expression of a variety of inflammatory molecules, exacerbating inflammation and tissue damage in SLE $(16,17)$. Oxidative damage mediated by ROS 
results in the generation of deleterious by-products, including aldehydic products, leading to the formation of highly immunogenic adducts with proteins. Thus, pathogenic antibodies are induced in a variety of diseases, including SLE and RA (18).

GSH is synthesized by two consecutive ATP-dependent enzymatic reactions. Glutamate cysteine ligase (GCL) catalyzes the first and rate-limiting step of GSH synthesis. GCL is a heterodimeric enzyme, consisting of a catalytic subunit, (GCLc) and a modulatory subunit (GCLm) that are encoded by two distinct genes (19). GCLc constitutes all the enzymatic activity, but catalytic efficiency is increased substantially by covalent interaction with GCLm $(20,21)$.

Although there is an association between SLE and GSH, GCL activity levels in immune cells from SLE patients remain unclear. Thus, the aim of the present study was to determine the changes in GCL activity in SLE. Thioredoxin (TRX) levels have been shown to increase in response to oxidative stress in experimental (22) and human studies (23). Thus, TRX concentrations were also examined in SLE patients and controls.

The SLE disease activity index (SLEDAI) is a validated model of global assessments from experienced clinicians on disease activity in lupus. It represents the consensus of a group of experts in the field of lupus study (24). Higher SLEDAI scores indicate a greater severity of disease (25). In addition, erythrocyte sedimentation rates (ESR) and autoantibodies are indicators of the degree of inflammation and may be used to monitor disease activity. Therefore, the associations between GSH levels and GCL activity with demographic characteristics, clinical manifestations and laboratory parameters in peripheral blood mononuclear cells (PBMCs) were analyzed in the present study.

\section{Materials and methods}

Patients and controls. A total of 30 SLE patients of Northern Han Chinese descent, diagnosed according to the criteria of the American College of Rheumatology, were enrolled in the study. Patients were selected from individuals attending outpatient meetings at the Department of Internal Medicine at Qingdao Municipal Hospital (Qingdao, China). In addition, 30 healthy controls were recruited that were ethnicity-, genderand age-matched with the patients. The patients enrolled were not smokers or alcoholics, and were not associated with any other autoimmune disease. Blood samples from the patients and healthy controls were collected with informed consent from the patients and approval from the Ethics Committee of Qingdao Municipal Hospital. Demographic, laboratory and clinical characteristics of the groups are listed in Table I.

Preparation of PBMCs. Peripheral blood samples (2 $\mathrm{ml})$, anticoagulated with sodium citrate, were collected from the controls and patients prior to the administration of any immunosuppressive drug. PBMCs were separated by density gradient centrifugation using the Ficoll Paque system (HengXin chemical reagent Co., Ltd., Shanghai, China), according to the manufacturer's instructions.

Laboratory measurements. For the patients with SLE, serum levels of C3, C4, IgG, IgA, IgM and C-reactive protein (CRP) were analyzed using an automatic nephelometric immuno- assay analyzer (Siemens, Munich, Germany). Autoantibodies, including antinuclear (ANA), anti-dsDNA and anti-Smith (Sm) antibodies, were detected using immunoblotting kits, according to the manufacturer's instructions (EUROIMMUN AG, Lübeck, Germany).

SLEDAI is a global score reflecting all aspects of disease activity and is a validated model for the assessment of disease activity in SLE (24). Disease activity was determined using the SLEDAI score. ESRs were analyzed using an automatic analyzer (Monitor-J+ analyzer, Vital Diagnostics Srl, Forli, Italy) in all the subjects.

Measurement of GSH and oxidized glutathione (GSSG) concentration in PBMCs. GSH concentration was measured using a GSH colorimetric assay kit (Beyotime Institute of Biotechnology, Shanghai, China), according to the manufacturer's instructions. PBMCs were prepared by density gradient centrifugation. The concentration was adjusted to $1 \times 10^{7}$ cells $/ \mathrm{ml}$ and the cells were washed in phosphate-buffered saline (PBS). This was followed by centrifugation at $10,000 \mathrm{x} \mathrm{g}$ at $4^{\circ} \mathrm{C}$ for $20 \mathrm{~min}$. Cells in the sediment were collected and mixed with protein removal solution $M(v / v, 1 / 3)$. The mixtures were vortexed fully and freeze-thawed twice using liquid nitrogen and a water bath. After placing on ice for $5 \mathrm{~min}$, the mixtures were centrifuged at $10,000 \times \mathrm{g}$ at $4^{\circ} \mathrm{C}$ for $10 \mathrm{~min}$. The supernatant was used for GSH detection. For the GSSG assay, GSH was removed with GSH removal liquid, following the manufacturer's instructions.

Analysis of TRX levels. PBMCs in PBS were adjusted to a concentration of $1 \times 10^{7}$ cells $/ \mathrm{ml}$ and freeze-thawed four times using liquid nitrogen and a water bath. The cells were then centrifuged at $5,000 \mathrm{x} \mathrm{g}$ at $4^{\circ} \mathrm{C}$ for $5 \mathrm{~min}$. The supernatant was used for TRX detection. TRX levels were analyzed using a human TRX ELISA assay kit (Uscn Life Science, Inc., Wuhan, China). Procedures were conducted according to the manufacturer's instructions.

GCL activity assay. GCL activity was determined by a fluorescence assay as described by Chen et al (26). The concentration of PBMCs was adjusted to $5 \times 10^{7}$ cells $/ \mathrm{ml}$ in TES/SB buffer (w/v, 1/4) consisting of $20 \mathrm{mM}$ Tris, $1 \mathrm{mM}$ EDTA, $250 \mathrm{mM}$ sucrose, $20 \mathrm{mM}$ sodium borate and $2 \mathrm{mM}$ serine. The cells were sonicated at $100 \mathrm{~W}$ for $60 \mathrm{sec}$ and then centrifuged at $10,000 \mathrm{x} \mathrm{g}$ at $4^{\circ} \mathrm{C}$ for $10 \mathrm{~min}$. The supernatants were collected and centrifuged again at $15,000 \mathrm{x} \mathrm{g}$ at $4^{\circ} \mathrm{C}$ for $20 \mathrm{~min}$. The supernatants were collected and the protein concentrations were determined using a bicinchoninic acid protein assay kit (Beyotime Institute of Biotechnology), with bovine serum albumin used as the standard.

For the GCL activity assay, aliquots of $30 \mu 1$ supernatant were mixed with $30 \mu \mathrm{l}$ GCL reaction cocktail (400 mM Tris, $40 \mathrm{mM}$ ATP, $40 \mathrm{mM}$ L-glutamic acid, 2 mM EDTA, $20 \mathrm{mM}$ sodium borate, $2 \mathrm{mM}$ serine and $40 \mathrm{mM} \mathrm{MgCl}$ ). Following incubation at $37^{\circ} \mathrm{C}$ for $5 \mathrm{~min}, 30 \mu \mathrm{l}$ cysteine solution $(30 \mathrm{mM}$; dissolved in TES/SB buffer) was added and the mixtures were incubated at $37^{\circ} \mathrm{C}$ for $13 \mathrm{~min}$. The enzymatic reaction in the mixtures was stopped by precipitating proteins with $200 \mathrm{mM}$ 5-sulfosalicylic acid (SSA). After placing on ice for $20 \mathrm{~min}$, the mixtures were centrifuged at $2,000 \mathrm{x} \mathrm{g}$ at $4^{\circ} \mathrm{C}$ for $10 \mathrm{~min}$. 
Following centrifugation, 20- $\mu 1$ samples of each supernatant containing $\gamma$-glutamylcysteine $(\gamma$-GC) were added to a 96 -well plate designed for fluorescence detection. For each assay, $20 \mu \mathrm{l}$ $\gamma$-GC standards, containing $5 \mu \mathrm{l}$ GCL reaction cocktail [5 $\mu 1$ SSA $(200 \mathrm{mM}), 5 \mu \mathrm{l} \mathrm{H}_{2} \mathrm{O}$ and $5 \mu \mathrm{l} \gamma$-GC standard solution $(0,20,40,60,80,100,120$ and $140 \mu \mathrm{M}$ in TES/SB buffer)], was added to each well of the same 96-well plate to generate a standard curve. Next, $180 \mu 1$ 2,3-naphthalenedicarboxyaldehyde (NDA) was added to each well. Following incubation in the dark at room temperature for $30 \mathrm{~min}$, the formation of NDA- $\gamma$-GC was measured (472 nm excitation/528 nm emission) using a fluorescent plate reader (GENios Plus; Tecan, Männedorf Switzerland). The production of $\gamma$-GC in each sample was calculated using the standard curve. Values were expressed in $\mathrm{mM}$ per min per $\mathrm{mg}$ of protein.

Statistical analysis. Statistical analysis was performed using SPSS software (version 13.0; SPSS, Inc., Chicago, IL, USA). Data are expressed as the mean \pm SD. The differences between the subject groups were analyzed using the independent Student's t-test, while correlation analysis was performed using Spearman's rank test. $\mathrm{P}<0.05$ was considered to indicate a statistically significant difference. Figures were constructed using GraphPad Prism software (version 5.0; GraphPad Software, Inc., La Jolla, CA, USA).

\section{Results}

Laboratory measurements of patients with SLE. Demographic characteristics, clinical manifestations and laboratory measurements of the patients with SLE are presented in Table I. Lupus nephritis (LN) is the major indicator of morbidity and mortality in SLE and was identified in 18 of the 30 patients. Arthritis, serositis and CNS disease were identified in 18, 9 and 2 patients, respectively.

A positive result for ANA, anti-dsDNA and anti-Sm autoantibodies was found in 30, 21 and 9 SLE patients, respectively. For the SLE patients, the mean ESR value was $54 \mathrm{~mm} / \mathrm{h}$, ranging between 8 and $119 \mathrm{~mm} / \mathrm{h}$. In addition, the mean CRP value was $47.2 \mathrm{mg} / \mathrm{l}$ with a range between 3 and $101 \mathrm{mg} / \mathrm{l}$. The mean SLEDAI value was 13.16, ranging between 2 and 39.

Levels of GSH and GSSG in PBMCs from patients with SLE and healthy controls. In order to explore the role of oxidative stress in SLE, GSH and GSSG concentrations and the redox state (GSH/GSSG), were examined in PBMCs from 30 patients with SLE and 30 gender- and age-matched healthy controls. GSH levels considerably decreased in PBMCs from the patients with SLE $(274.90 \pm 17.08 \mathrm{nmol} / \mathrm{mg}$ protein $)$ compared with those in the healthy controls $(413.63 \pm 20.79 \mathrm{nmol} / \mathrm{mg}$ protein; $\mathrm{P}<0.0001$; Fig. 1). By contrast, GSSG levels significantly increased $(124.95 \pm 4.27 \mathrm{nmol} / \mathrm{mg}$ protein; $\mathrm{P}<0.01)$ in patients with SLE compared with those in the healthy controls $(68.94 \pm 1.89 \mathrm{nmol} / \mathrm{mg}$ protein; Table II). The reduction in GSH and increase in GSSG concentrations resulted in a decreased redox state (GSH/GSSG) in patients with SLE (2.27 \pm 0.43 ; $\mathrm{P}<0.001)$ compared with that in the healthy controls $(6.11 \pm 1.07$; Table II). Furthermore, the levels of the antioxidant GSH were markedly decreased in patients with SLE and LN $(243.33 \pm 16.73 \mathrm{nmol} / \mathrm{mg}$ protein) compared with those in
Table I. Demographic characteristics, clinical features and laboratory measurements of the subjects ( $n=30$ per group).

\begin{tabular}{lcc}
\hline & $\begin{array}{c}\text { SLE } \\
\text { patients }\end{array}$ & $\begin{array}{c}\text { Healthy } \\
\text { controls }\end{array}$ \\
\hline Demaracteristics & & \\
Female, n (\%) & $24(80)$ & $23(76.7)$ \\
Male, n (\%) & $6(20)$ & $7(23.3)$ \\
Age, years & $34.7(21-57)$ & $35.5(22-60)$ \\
Clinical features & & \\
LN, n (\%) & $18(60)$ & - \\
Arthritis, n (\%) & $18(60)$ & - \\
Serositis, n (\%) & $9(30)$ & - \\
CNS disease, n (\%) & $2(6.7)$ & - \\
SLEDAI & $13.16(2-39)$ & - \\
Laboratory measurements & & - \\
C3, g/l & $1.10(0.87-1.7)$ & - \\
C4, g/l & $0.073(0.07-0.48)$ & - \\
IgG, g/l & $16.94(6.8-34.7)$ & - \\
IgA, g/l & $1.49(0.68-3.35)$ & - \\
IgM, g/l & $1.38(0.32-2.12)$ & - \\
ANA, n (\%) & $30(100)$ & - \\
Anti-dsDNA, n (\%) & $21(70)$ & - \\
Anti-Sm, n (\%) & $9(30)$ & - \\
CRP, mg/l & $57.2(3-101)$ & - \\
ESR, mm/h & $54(8-119)$ & - \\
\hline
\end{tabular}

Except where indicated otherwise, values are the mean (range). SLE, systemic lupus erythematosus; LN, lupus nephritis; CNS, central nervous system; SLEDAI, systemic lupus erythematosus disease activity index; ANA, antinuclear antibody; anti-Sm, anti-Smith antibody; CRP, C-reactive protein; ESR, erythrocyte sedimentation rate.

SLE patients without LN $(322.25 \pm 30.58 \mathrm{nmol} / \mathrm{mg}$ protein; $\mathrm{P}<0.02$; Fig. 2). The level of GSSG significantly increased $(121.06 \pm 8.32 \mathrm{nmol} / \mathrm{mg}$ protein; $\mathrm{P}<0.05)$ and the redox state markedly decreased $(2.01 \pm 0.58 ; \mathrm{P}<0.05)$ in patients with SLE and LN compared with those in SLE patients without LN (Table II).

TRX levels in PBMCs from patients with SLE and healthy controls. In order to further investigate the status of oxidative stress in SLE, TRX concentrations in the PBMCs from patients with SLE were examined. The average TRX concentration in the patients with SLE was $27.2 \pm 9.7 \mathrm{ng} / \mathrm{ml}$, which was significantly increased compared with that in the healthy controls (14.6 $\pm 7.2 ; \mathrm{P}<0.01$; Table II). In addition, the concentration of TRX significantly increased $(34.2 \pm 5.6 ; \mathrm{P}<0.05)$ in patients with SLE and LN when compared with the concentration in SLE patients without LN (Table II).

Changes in the enzymatic activity levels of GCL in PBMCs from patients with SLE and healthy controls. GCL activity levels in PBMCs from the 30 SLE patients were analyzed. The average GCL activity level in the patients with SLE was $266.10 \pm 13.31 \mathrm{mmol} / \mathrm{min} / \mathrm{mg}$ protein, which was signifi- 
Table II. Oxidant and antioxidant parameters in PBMCs from patients with SLE and healthy controls.

\begin{tabular}{lcccc}
\hline Parameters & Controls & SLE patients & SLE patients with LN & SLE patients without LN \\
\hline GSH, nmol/mg protein & $413.63 \pm 20.79$ & $274.90 \pm 17.08^{\mathrm{c}}$ & $243.33 \pm 16.73$ & $322.25 \pm 30.58^{\mathrm{a}}$ \\
GSSG, nmol/mg protein & $68.94 \pm 1.89$ & $124.95 \pm 4.27^{\mathrm{b}}$ & $121.06 \pm 8.32$ & $147.15 \pm 7.51^{\mathrm{a}}$ \\
GSH/GSSG & $6.11 \pm 1.07$ & $2.27 \pm 0.43^{\mathrm{c}}$ & $2.01 \pm 0.58$ & $2.29 \pm 0.51^{\mathrm{a}}$ \\
TRX, ng/ml & $14.6 \pm 7.2$ & $27.2 \pm 9.7^{\mathrm{b}}$ & $34.2 \pm 5.6$ & $25.7 \pm 6.3^{\mathrm{a}}$ \\
\hline
\end{tabular}

Values are expressed as mean $\pm \mathrm{SD}$. ${ }^{\mathrm{P}} \mathrm{P}<0.05$, vs. SLE patients with $\mathrm{LN} ;{ }^{\mathrm{b}} \mathrm{P}<0.01$ and ${ }^{\mathrm{c}} \mathrm{P}<0.001$, vs. control. PBMCs, peripheral blood mononuclear cells; SLE, systemic lupus erythematosus; GSH, glutathione; GSSG, oxidized glutathione; TRX, thioredoxin; LN, lupus nephritis.

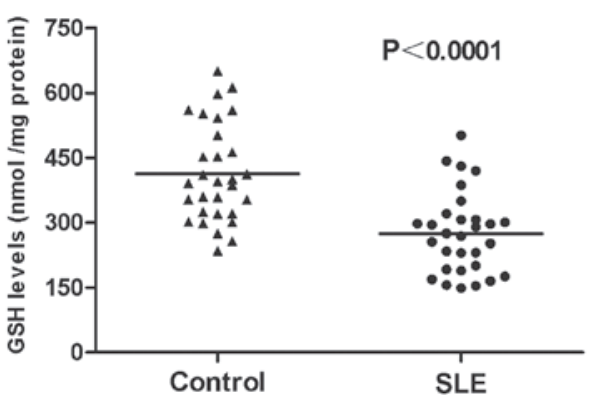

Figure 1. GSH levels in PBMCs from patients with SLE $(\mathrm{n}=30)$ and controls $(\mathrm{n}=30)$. Horizontal lines indicate mean values (SLE patients, 274.9; controls, $413.6 \mathrm{nmol} / \mathrm{mg}$ protein). There was a significant reduction in GSH levels in the SLE patients compared with the controls $(\mathrm{P}<0.0001)$. GSH, glutathione; PBMCs, peripheral blood mononuclear cells; SLE, systemic lupus erythematosus.

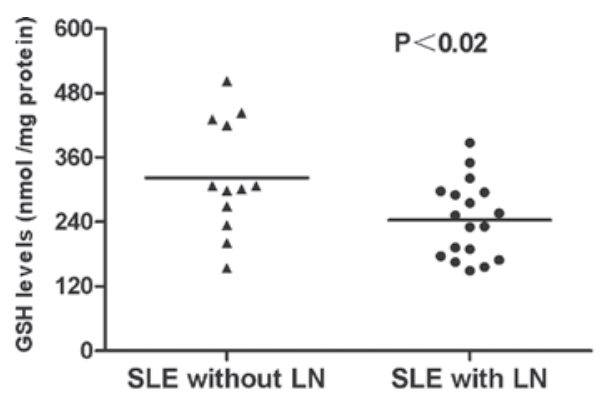

Figure 2. GSH levels in PBMCs from patients with SLE, with $(n=18)$ and without $(n=12)$ LN. Horizontal lines indicate mean values (SLE patients with LN, 243.3; SLE patients without LN, $322.2 \mathrm{nmol} / \mathrm{mg}$ protein). There was a significant reduction in GSH levels in the SLE patients with LN compared with those without LN $(\mathrm{P}<0.02)$. GSH, glutathione; PBMCs, peripheral blood mononuclear cells; SLE, systemic lupus erythematosus; LN, lupus nephritis.

cantly reduced compared with that in the healthy controls (383.27 $\pm 18.68 ; \mathrm{P}<0.0001$; Fig. 3). In addition, GCL activity levels in SLE patients with LN (229.64+17.82) were significantly lower when compared with those in the SLE patients without LN (303.25 \pm 18.47 ; $\mathrm{P}<0.009$; Fig. 4).

Correlation analysis between GSH levels and characteristics or laboratory parameters in patients with SLE. Associations between GSH levels in PBMCs and demographic characteristics, clinical manifestations and laboratory parameters were analyzed. The results demonstrated that the levels of GSH in the PBMCs negatively correlated with SLEDAI

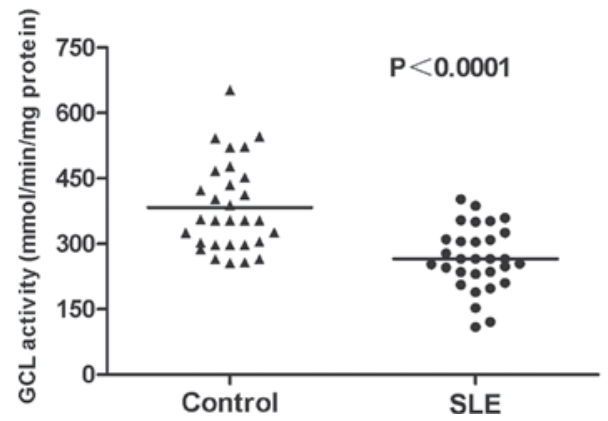

Figure 3. GCL activity levels in PBMCs from patients with SLE $(n=30)$ and controls $(n=30)$. Horizontal lines indicate mean values (SLE patients, 266.1; controls, $383.2 \mathrm{mmol} / \mathrm{min} / \mathrm{mg}$ protein). There was a significant reduction in GCL activity levels in patients with SLE compared with the controls $(\mathrm{P}<0.0001)$. GCL, glutamate cysteine ligase; PBMCs, peripheral blood mononuclear cells; SLE, systemic lupus erythematosus.

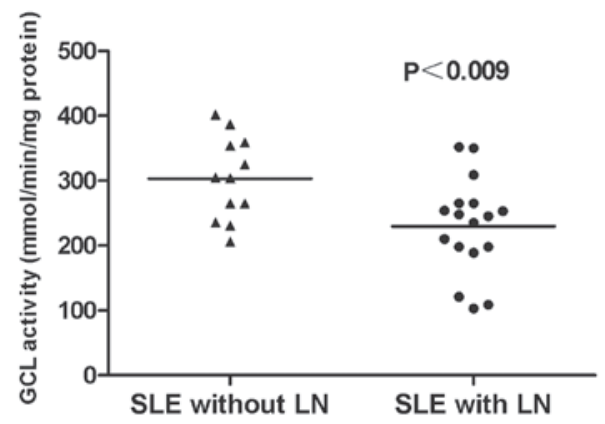

Figure 4. GCL activity levels in PBMCs from patients with SLE, with (n=18) and without $(n=12)$ LN. Horizontal lines indicate mean values (SLE patients with LN, 229.6; SLE patients without LN, $303.2 \mathrm{mmol} / \mathrm{min} / \mathrm{mg}$ protein). There was a significant reduction in GCL activity levels in SLE patients with LN compared with those without LN $(\mathrm{P}<0.009)$. GCL, glutamate cysteine ligase; PBMCs, peripheral blood mononuclear cells; SLE, systemic lupus erythematosus; LN, lupus nephritis.

values ( $\mathrm{r}=-0.565 ; \mathrm{P}<0.001$; Fig. 5). No statistically significant associations were identified between GSH levels and other characteristics, clinical manifestations or laboratory parameters in the patients with SLE.

Correlation analysis between GCL activity and characteristics or laboratory parameters in patients with SLE. Associations between GCL activity levels in PBMCs and demographic characteristics, clinical manifestations and laboratory parameters were analyzed. The results revealed that GCL activity levels in PBMCs negatively correlated with SLEDAI scores 


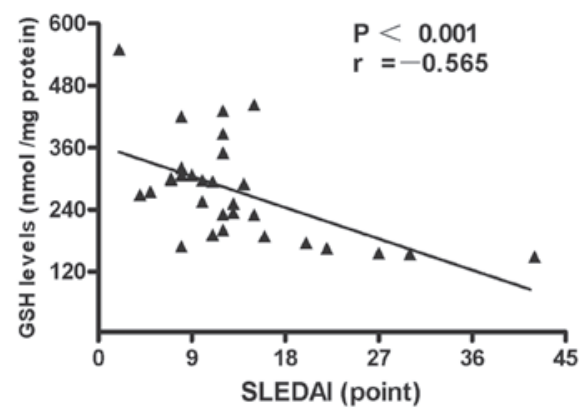

Figure 5. Negative correlation between GSH levels and SLEDAI values in patients with SLE $(n=30)$. SLE, systemic lupus erythematosus; GSH, glutathione; SLEDAI, systemic lupus erythematosus disease activity index.

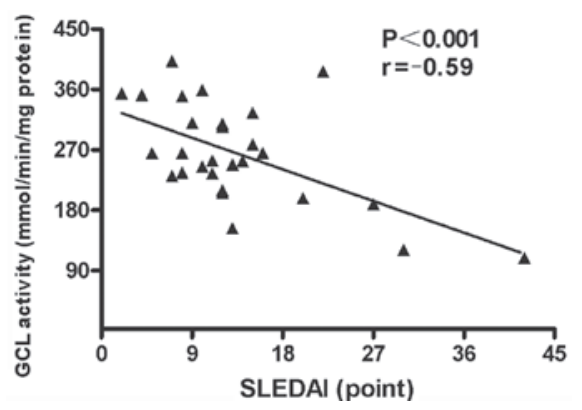

Figure 6. Negative correlation between GCL activity levels and SLEDAI values in patients with SLE $(\mathrm{n}=30)$. SLE, systemic lupus erythematosus; GCL, glutamate cysteine ligase; SLEDAI, systemic lupus erythematosus disease activity index.

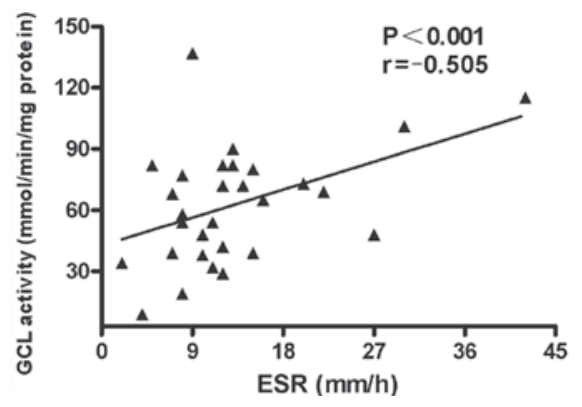

Figure 7. Negative correlation between GCL activity levels and ESRs in patients with SLE $(n=30)$. SLE, systemic lupus erythematosus; GCL, glutamate cysteine ligase; ESR, erythrocyte sedimentation rate.

$(\mathrm{r}=-0.59 ; \mathrm{P}<0.001 ;$ Fig. 6$)$ and ESR $(\mathrm{r}=-0.505 ; \mathrm{P}<0.001 ;$ Fig. 7$)$ in patients with SLE. No statistically significant correlations were identified between GCL activity levels and other characteristics, clinical manifestations or laboratory parameters in the patients with SLE.

\section{Discussion}

Oxidative stress is hypothesized to play a major role in the initiation and progression of autoimmune disease by excessive free radical formation (27). The inability of the antioxidant defense system to cope with oxidative stress is considered to be a possible cause in SLE. The inflammatory nature of the disease indicates that excessive ROS production and an imbalanced redox system may contribute to the immune dysfunction, autoantigen production and perturbation of programmed cell death in SLE (28).

In the present study, GCL activity levels in SLE patients were analyzed, and negative correlations between GCL activity and SLEDAI or ESR were identified. The results clearly indicate that GSH levels and GCL enzymatic activity levels decrease significantly in the PBMCs of patients with SLE, which may be a hallmark of oxidative stress in SLE and coupled together as a cause and consequence in the severity of the disease. The results indicate that GCL activity levels may be involved in disease activity and pathogenesis of SLE.

GCL catalyzes the first and rate-limiting step of GSH synthesis, in which glutamate ligates with cysteine to form $\gamma$-GC. This rapidly reacts with glycine to form GSH via the action of GSH synthase (29). The present study demonstrated a good correlation between GSH levels and GCL activity, the primary determinant of the rate of GSH synthesis (30). These observations indicate that a reduction in GCL activity results in a reduction in GSH levels in SLE patients. Consistent with the reduction in GSH levels, GCL activity was inadequate in the PBMCs of patients with SLE. The deficiency of GCL activity and reduction in GSH concentration may contribute to the pathogenesis of SLE through several mechanisms. One possible mechanism is that insufficient GSH content may affect caspase activity, transcription factor activation, Bcl-2 expression and function, thiol-redox signaling and phosphatidylserine externalization, which are early processes in apoptosis $(28,31)$. A negative association of GSH levels with T-lymphocyte and $\mathrm{CD} 4^{+}$and $\mathrm{CD} 8^{+}$lymphocyte subset apoptosis, and intracellular activated caspase-3 may support the role of GSH in the alteration of apoptosis of T lymphocytes in the SLE disease state (1). These results indicate that GSH is involved in the depletion of $\mathrm{CD} 4^{+} \mathrm{T}$ lymphocytes in patients with SLE (1). The reduction in cellular GSH levels has been attributed mainly to GSH oxidation, promoted by increased production of ROS in the cells (32). It has been reported that GSH depletion in antigen-presenting cells inhibits the production of Th1-related cytokines, including interferon- $\gamma$ and interleukin-12, and supports the Th2-mediated humoral immune response (33). Since GSH has a significant effect on the ability of the immune system to activate the appropriate Th response, altering the levels of GSH may have significant implications in Th1/Th2-related diseases, including SLE (26). Consequently, insufficient levels of GCL activity and GSH may result in SLE disease, indicating a critical and cell-specific function in the etiology of SLE.

Adequate concentrations of GSH are required for a variety of functions, including the protection of the cell from oxidative damage, the quenching of oxidant species, lymphocyte activation, natural killer cell activation and lymphocyte-mediated cytotoxicity $(34,35)$. A reduction in the level of intracellular GSH correlates with the severity of disease, particularly in patients with $\mathrm{LN}(36,37)$.

The inverse correlation between GCL activity, GSH and SLE disease activity and severity, indicated by SLEDAI scores and ESRs, indicates that insufficient levels of GCL activity and GSH may contribute to the severity of disease. Higher SLEDAI scores indicate more severe disease activity (25). Thus, the negative correlation between GCL activity, GSH and SLEDAI scores in patients with SLE indicates that the lower the levels of GCL activity and GSH, the more severe 
the disease. In addition, ESR is an indicator of the degree of inflammation and is used to monitor disease activity. Since GCL activity was shown to negatively correlate with the ESR in patients with SLE, GCL activity levels may be an index of disease activity. In addition, there was a significant difference in GCL activity and GSH levels between SLE patients with and without LN. Therefore, correlation analysis between GCL activity and SLEDAI, ESR and LN further indicates a potential role of GCL activity and GSH in the pathogenesis of SLE.

TRX is elevated in patients with increased oxidative stress, including AIDS (38) and RA (39). In the present study, increased TRX levels were observed in patients with SLE, which further demonstrates the change in redox state in SLE patients.

In conclusion, GCL enzymatic activity is downregulated and inversely correlates with specific disease parameters in SLE patients. The results indicate that a reduction in GCL activity levels correlates with SLE disease activity and severity. The results support the hypothesis that oxidative stress is a therapeutic target for pharmacological agents in SLE. However, further mechanistic in vitro and in vivo studies are required to investigate how the interplay between GSH and pathogenesis may lead to the intolerance and aggressiveness of SLE disease activity. Further studies should be directed to evaluate the role of GSH in the pathogenesis of SLE.

\section{References}

1. Shah D, Aggarwal A, Bhatnagar A, et al: Association between $\mathrm{T}$ lymphocyte sub-sets apoptosis and peripheral blood mononuclear cells oxidative stress in systemic lupus erythematosus. Free Radic Res 45: 559-567, 2011.

2. Gordon C: Long-term complications of systemic lupus erythematosus. Rheumatology (Oxford) 41: 1095-1100, 2002.

3. Mansour RB, Lassoued S, Gargouri B, et al: Increased levels of autoantibodies against catalase and superoxide dismutase associated with oxidative stress in patients with rheumatoid arthritis and systemic lupus erythematosus. Scand J Rheumatol 37 : 103-108, 2008

4. Kurien BT and Scofield RH: Free radical mediated peroxidative damage in systemic lupus erythematosus. Life Sci 73: 1655-1666, 2003.

5. Munoz LE, Gaipl US and Herrmann M: Predictive value of anti-dsDNA autoantibodies: importance of the assay. Autoimmun Rev 7: 594-597, 2008.

6. Li J, Ayene R, Ward KM, et al: Glucose deprivation increases nuclear DNA repair protein $\mathrm{Ku}$ and resistance to radiation induced oxidative stress in human cancer cells. Cell Biochem Funct 27: 93-101, 2009.

7. Townsend DM, Tew KD and Tapiero $\mathrm{H}$ : The importance of glutathione in human disease. Biomed Pharmacother 57: 145-155, 2003

8. Gambhir JK, Lali P and Jain AK: Correlation between blood antioxidant levels and lipid peroxidation in rheumatoid arthritis. Clin Biochem 30: 351-355, 1997.

9. Pastore A, Federici G, Bertini E and Piemonte F: Analysis of glutathione: implication in redox and detoxification. Clin Chim Acta 333: 19-39, 2003.

10. Burek CL and Rose NR: Autoimmune thyroiditis and ROS. Autoimmun Rev 7: 530-537, 2008.

11. Griffiths HR: Is the generation of neo-antigenic determinants by free radicals central to the development of autoimmune rheumatoid disease? Autoimmun Rev 7: 544-549, 2008.

12. Perricone C, De Carolis C and Perricone R: Glutathione: a key player in autoimmunity. Autoimmun Rev 8: 697-701, 2009.

13. Fernandez D and Perl A: Metabolic control of T cell activation and death in SLE. Autoimmun Rev 8: 184-189, 2009.

14. Dimitrov JD, Vassilev TL, Andre S, et al: Functional variability of antibodies upon oxidative processes. Autoimmun Rev 7 : 574-578, 2008

15. Crane FL and Low H: Reactive oxygen species generation at the plasma membrane for antibody control. Autoimmun Rev 7: 518-522, 2008
16. Grisham MB: Reactive oxygen species in immune responses. Free Radic Biol Med 36: 1479-1480, 2004.

17. Hassan SZ, Gheita TA, Kenawy SA, et al: Oxidative stress in systemic lupus erythematosus and rheumatoid arthritis patients: relationship to disease manifestations and activity. Int J Rheum Dis 14: 325-331, 2011.

18. Kurien BT and Scofield RH: Autoimmunity and oxidatively modified autoantigens. Autoimmun Rev 7: 567-573, 2008.

19. Huang CS, Chang LS, Anderson ME and Meister A: Catalytic and regulatory properties of the heavy subunit of rat kidney gamma-glutamylcysteine synthetase. J Biol Chem 268: 19675-19680, 1993.

20. McConnachie LA, Mohar I, Hudson FN, et al: Glutamate cysteine ligase modifier subunit deficiency and gender as determinants of acetaminophen-induced hepatotoxicity in mice. Toxicol Sci 99: 628-636, 2007.

21. Dalton TP, Dieter MZ, Yang Y, et al: Knockout of the mouse glutamate cysteine ligase catalytic subunit (Gclc) gene: Embryonic lethal when homozygous, and proposed model for moderate glutathione deficiency when heterozygous. Biochem Biophys Res Commun 279: 324-329, 2000.

22. Kasuno K, Nakamura H, Ono T, et al: Protective roles of thioredoxin, a redox-regulating protein, in renal ischemia/reperfusion injury. Kidney Int 64: 1273-1282, 2003.

23. Maurice MM, Nakamura H, Gringhuis S, et al: Expression of the thioredoxin-thioredoxin reductase system in the inflamed joints of patients with rheumatoid arthritis. Arthritis Rheum 42: 2430-2439, 1999.

24. Bombardier C, Gladman DD, Urowitz MB, et al: Derivation of the SLEDAI. A disease activity index for lupus patients. The Committee on Prognosis Studies in SLE. Arthritis Rheum 35: 630-640, 1992.

25. Hawker G, Gabriel S, Bombardier C, et al: A reliability study of SLEDAI: a disease activity index for systemic lupus erythematosus. J Rheumatol 20: 657-660, 1993.

26. Chen CN, Brown-Borg HM, Rakoczy SG, et al: Aging impairs the expression of the catalytic subunit of glutamate cysteine ligase in soleus muscle under stress. J Gerontol A Biol Sci Med Sci 65: 129-137, 2010.

27. Shah D, Sah S and Nath SK: Interaction between glutathione and apoptosis in systemic lupus erythematosus. Autoimmun Rev 12: 741-751, 2013.

28. Ortona E, Margutti P, Matarrese P, et al: Redox state, cell death and autoimmune diseases: a gender perspective. Autoimmun Rev 7: 579-584, 2008.

29. Ziegler DM: Role of reversible oxidation-reduction of enzyme thiols-disulfides in metabolic regulation. Annu Rev Biochem 54: 305-329, 1985.

30. Maher P: The effects of stress and aging on glutathione metabolism. Ageing Res Rev 4: 288-314, 2005.

31. Kasahara Y,Iwai K, Yachie A, et al: Involvement of reactive oxygen intermediates in spontaneous and CD95 (Fas/APO-1)-mediated apoptosis of neutrophils. Blood 89: 1748-1753, 1997.

32. Hammond CL, Madejczyk MS and Ballatori N: Activation of plasma membrane reduced glutathione transport in death receptor apoptosis of HepG2 cells. Toxicol Appl Pharmacol 195: $12-22,2004$.

33. Messina JP and Lawrence DA: Cell cycle progression of glutathione-depleted human peripheral blood mononuclear cells is inhibited at S phase. J Immunol 143: 1974-1981, 1989.

34. Franco R, Panayiotidis MI and Cidlowski JA: Glutathione depletion is necessary for apoptosis in lymphoid cells independent of reactive oxygen species formation. J Biol Chem 282: 30452-30465, 2007.

35. Ballatori N, Krance SM, Notenboom S, et al: Glutathione dysregulation and the etiology and progression of human diseases. Biol Chem 390: 191-214, 2009.

36. Shah D, Kiran R, Wanchu A and Bhatnagar A: Oxidative stress in systemic lupus erythematosus: relationship to Th1 cytokine and disease activity. Immunol Lett 129: 7-12, 2010.

37. Túri S, Németh I, Torkos A, et al: Oxidative stress and antioxidant defense mechanism in glomerular diseases. Free Radic Biol Med 22: 161-168, 1997.

38. Nakamura H, De Rosa SC, Yodoi J, et al: Chronic elevation of plasma thioredoxin: inhibition of chemotaxis and curtailment of life expectancy in AIDS. Proc Natl Acad Sci USA 98: 2688-2693, 2001.

39. Jikimoto T, Nishikubo Y, Koshiba M, et al: Thioredoxin as a biomarker for oxidative stress in patients with rheumatoid arthritis. Mol Immunol 38: 765-772, 2002. 